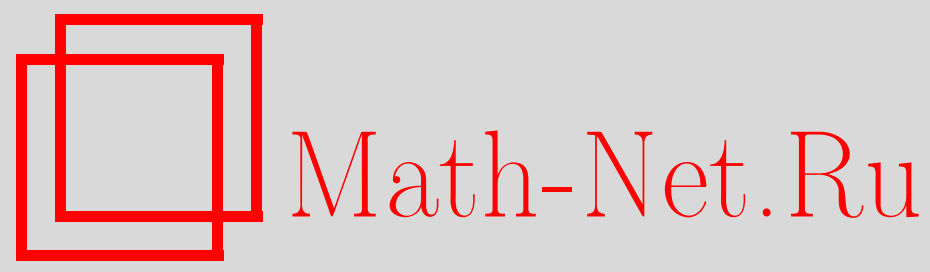

А. И. Звягинцев, О точных неравенствах для производных функций, удовлетворяющих краевым условиям, $M a-$ тем. заметки, 1997, том 62, выпуск 5, 712-724

DOI: https://doi.org/10.4213/mzm1658

Использование Общероссийского математического портала Math-Net.Ru подразумевает, что вы прочитали и согласны с пользовательским соглашением http://www.mathnet.ru/rus/agreement

Параметры загрузки:

IP : 54.198 .64 .247

26 апреля 2023 г., 13:18:00 


\section{О ТОЧНЫХ НЕРАВЕНСТВАХ ДЛЯ ПРОИЗВОДНЫХ ФУНКЦИЙ, УДОВЛЕТВОРЯЮЩИХ КРАЕВЫМ УСЛОВИЯМ}

\section{А. И. Звягинцев}

Для функций, удовлетворяющих краевым условиям

$$
f(0)=f^{\prime}(0)=\cdots=f^{(m)}(0)=0, \quad f(1)=f^{\prime}(1)=\cdots=f^{(l)}(1)=0,
$$

доказывается неравенство с точными константами в аддитивной форме

$$
\left\|f^{(n-1)}\right\|_{L_{q}(0,1)} \leqslant A\|f\|_{L_{p}(0,1)}+B\left\|f^{(n)}\right\|_{L_{r}(0,1)},
$$

где $n \geqslant 2,0 \leqslant l \leqslant n-2,-1 \leqslant m \leqslant l, m+l \leqslant n-3,1 \leqslant p, q, r \leqslant \infty$.

Библиография: 22 названия.

1. Задача о точных константах. Пусть функция $f(x)$ одной переменной задана на конечном отрезке и имеет абсолютно непрерьвную производную $f^{(n-1)}$ на этом отрезке. Не уменьшая общности, в качестве конечного отрезка возьмем [0,1]. Хорошо известно, что для любых целых $k$ и $n, 0 \leqslant k<n$, и любых $1 \leqslant p, q, r \leqslant \infty$ сушествуют постоянные $A$ и $B$ такие, что

$$
\left\|f^{(k)}\right\|_{q} \leqslant A\|f\|_{p}+B\left\|f^{(n)}\right\|_{r}
$$

где $\|\cdot\|_{p}=\|\cdot\|_{L_{p}(0,1)}$.

Рассмотрим неравенство (1) для функций, удовлетворяющих краевым условиям

$$
\begin{gathered}
f(0)=f^{\prime}(0)=\cdots=f^{(m)}(0)=0, \\
f(1)=f^{\prime}(1)=\cdots=f^{(l)}(1)=0,
\end{gathered}
$$

где $-1 \leqslant m \leqslant n-1,-1 \leqslant l \leqslant n-1, m+l \leqslant n-2$ (в случае $m=-1$ или $l=-1$ предполагается отсутствие соответственно условий (2) или (3)).

Положим

$$
A_{n, k, m, l}^{*} \equiv A_{n, k, m, l}^{*}(p, q, r)=\inf A,
$$

где инфимум берется по всем парам $(A, B)$, для которых имеет место $(1)$, и

$$
B_{n, k, m, l}^{*} \equiv B_{n, k, m, l}^{*}(p, q, r)=\inf B,
$$

Работа выполнена при финансовой поддержке Российского фонда фундаментальных исследований, грант № 93-011-255. 
где инфимум берется уже по парам $\left(A_{n, k, m, l}^{*}, B\right)$, для которых также вьполнено $(1)$.

В некоторых частных случаях значения и оценки точных констант $A_{n, k, m, l}^{*}, B_{n, k, m, l}^{*}$ получены в работах [1]-[20]. Следует отметить, что наиболее исследованным является случай $m=l=-1$.

Рассмотрим для функций, удовлетворяющих условиям (2) и (3), неравенство

$$
\left\|f^{(n-1)}\right\|_{q} \leqslant A_{n, n-1, m, l}^{*}\|f\|_{p}+B_{n, n-1, m, l}^{*}\left\|f^{(n)}\right\|_{r} .
$$

Замена $x$ на $1-x$ позволяет ограничиться изучением только случая $l \geqslant m$. В дальнейшем полагаем, что $l \geqslant m$.

Если $l=m=-1$, то, как показано В.И. Буренковьп [1], [2], для $n \geqslant 1,1 \leqslant p, q, r \leqslant \infty$ $A_{n, n-1,-1,-1}^{*}=\frac{(n-1) !}{\left\|Q_{n-1, p}\right\|_{p}}, \quad \frac{1}{2}\left((1+q)^{-1 / q}-\frac{1}{n}\right) \leqslant B_{n, n-1,-1,-1}^{*} \leqslant 2^{(1 / r-1 / q)_{+}-1}$, где $Q_{n-1, p}(x)=x^{n-1}+a_{n-2} x^{n-2}+\cdots+a_{0}$ - многочлен, наименее уклоняющийся от нуля в метрике $L_{p}(0,1)$, и $c_{+}=\max \{c, 0\}$.

Если $l=n-1$, то из результатов Д. Бойда [11] следует, что для $n \geqslant 1,1 \leqslant p, q, r \leqslant \infty$

$$
\begin{gathered}
A_{n, n-1,-1, n-1}^{*}=0, \\
B_{n, n-1,-1, n-1}^{*}=\left(\frac{r q^{q}}{(r-1) q}\right)^{1 / q}\left(\frac{(r-1) q+r}{(r-1) q}\right)^{1 / r-1 / q} \mathrm{~B}^{-1}\left(\frac{1}{q}, 1-\frac{1}{r}\right),
\end{gathered}
$$

где $\mathrm{B}(u, v)$ - бэта-функция.

Мы рассматриваем случай

$$
n \in\{2,3, \ldots,\}, \quad 0 \leqslant l \leqslant n-2, \quad-1 \leqslant m \leqslant l, \quad m+l \leqslant n-3 .
$$

Обозначим через $Q_{n-1, p, m, l}(x)$ многочлен степени $n-1$, удовлетворяющий условиям $(2),(3)$ и наименее уклоняющийся от нуля в метрике $L_{p}(0,1)$, т.е.

$$
Q_{n-1, p, m, l}(x)=\left\{\begin{array}{l}
x^{m+1}(x-1)^{l+1} \text { при } m+l=n-3, \\
x^{m+1}(x-1)^{l+1}\left(x^{n-m-l-3}+a_{n-m-l-4} x^{n-m-l-4}+\cdots+a_{0}\right) \\
\text { при } m+l \leqslant n-4,
\end{array}\right.
$$

причем

$$
\left\|Q_{n-1, p, m, l}\right\|_{p}=\inf _{b \in \mathbb{R}^{n-m-l-3}} \Phi_{p}(b)
$$

где $b=\left(b_{0}, \ldots, b_{n-m-l-4}\right)$ и

$$
\Phi_{p}(b)=\left\|x^{m+1}(x-1)^{l+1}\left(x^{n-m-l-3}+b_{n-m-l-4} x^{n-m-l-4}+\cdots+b_{0}\right)\right\|_{p} .
$$

Существование $Q_{n-1, p, m, l}$ при $1 \leqslant p \leqslant \infty$ следует из того, что функция $\Phi_{p}(b)$ является выпуклой, непрерывной по $b$ и неограниченно возрастает при $b \rightarrow \infty$.

Отметим, что многочлен $Q_{n-1, p, m, l}$ при $1 \leqslant p<\infty$ и $m+l \leqslant n-4$ удовлетворяет системе уравнений

$$
\int_{0}^{1} t^{i+m+1}(t-1)^{l+1}\left|Q_{n-1, p, m, l}(t)\right|^{p-1} \operatorname{sign} Q_{n-1, p, m, l}(t) d t=0, \quad i=0, \ldots, n-m-l-4 .
$$


Tеорема 1. Пусть $1 \leqslant p, q, r \leqslant \infty$ и выполняются условия (5). Тогда для функиий, удовлетворяющих условиям (2) и (3), в неравенстве (4) для точных констант выполнены соотношения

$$
A_{n, n-1, m, l}^{*}=\frac{(n-1) !}{\left\|Q_{n-1, p, m, l}\right\|_{p}}, \quad \frac{1}{2}\left((1+q)^{-1 / q}-\frac{1}{n}\right) \leqslant B_{n, n-1, m, l}^{*} \leqslant 1 .
$$

ДокАЗАТЕЛЬСтво. Для первой константы в неравенстве (4) при $1 \leqslant p \leqslant \infty$ справедлива очевидная оценка снизу

$$
\frac{(n-1) !}{\left\|Q_{n-1, p, m, l}\right\|_{p}} \leqslant A_{n, n-1, m, l}^{*}
$$

так как в противном случае для $f=Q_{n-1, p, m, l}$ в (4) получается противоречие.

Рассмотрим сначала случай $1<p<\infty$. Воспользуемся интегральньп представлением [1], [2]

$$
f^{(n-1)}(x)=\int_{0}^{1} \omega(\xi) f^{(n-1)}(\xi) d \xi+\int_{0}^{1} \Lambda(x, \xi) f^{(n)}(\xi) d \xi,
$$

где функция $\omega(\xi)$ имеет на $[0,1]$ абсолютно непрерьвную производную $\omega^{(n-1)}(\xi)$ и

$$
\begin{gathered}
\omega(0)=\cdots=\omega^{(n-m-3)}(0)=0, \\
\omega(1)=\cdots=\omega^{(n-l-3)}(1)=0, \\
\int_{0}^{1} \omega(\xi) d \xi=1, \\
\Lambda(x, \xi)= \begin{cases}\int_{0}^{\xi} \omega(\eta) d \eta, & 0 \leqslant \xi \leqslant x, \\
-\int_{\xi}^{1} \omega(\eta) d \eta, & x<\xi \leqslant 1 .\end{cases}
\end{gathered}
$$

Отметим, что в случае $m=-1$ и $l=n-2$ условия (10) отсутствуют, а $n-m-3 \geqslant 0$ в силу (5).

Интегрируя в (8) по частям с учетом (2), (3), (9), (10), получим

$$
f^{(n-1)}=A_{\omega} f+B_{\omega} f^{(n)},
$$

где

$$
\left(A_{\omega} \varphi\right)(x)=(-1)^{n-1} \int_{0}^{1} \omega^{(n-1)}(\xi) \varphi(\xi) d \xi, \quad\left(B_{\omega} \varphi\right)(x)=\int_{0}^{1} \Lambda(x, \xi) \varphi(\xi) d \xi .
$$

Из (13) следует, что

$$
\left\|f^{(n-1)}\right\|_{q} \leqslant\left\|A_{\omega}\right\|_{L_{p} \rightarrow L_{q}}\|f\|_{p}+\left\|B_{\omega}\right\|_{L_{r} \rightarrow L_{q}}\left\|f^{(n)}\right\|_{r} .
$$

Выберем функцию $\omega(\xi)$, удовлетворяющую перечисленным выше условиям так, чтобы норма

$$
\left\|A_{\omega}\right\|_{L_{p} \rightarrow L_{q}}=\left\|\omega^{(n-1)}\right\|_{p^{\prime}}
$$


была минимальна. Для этого, решая уравнение Эйлера в задаче на условный экстремум

$$
\int_{0}^{1}\left|\omega^{(n-1)}(\xi)\right|^{p^{\prime}} d \xi \rightarrow \inf , \quad \int_{0}^{1} \omega(\xi) d \xi=1
$$

получим [1]

$$
\omega^{(n-1)}(\xi)=\alpha\left|P_{n-1}(\xi)\right|^{p-1} \operatorname{sign} P_{n-1}(\xi),
$$

где $\alpha \neq 0$ и $P_{n-1}(\xi)$ - многочлен степени $\leqslant n-1$.

По формуле Тейлора с учетом (9) имеем

$$
\omega(t)=\frac{1}{(n-m-3) !} \int_{0}^{t}(t-\eta)^{n-m-3} \omega^{(n-m-2)}(\eta) d \eta .
$$

При $0 \leqslant l \leqslant n-3$ (т.е. исключается случай $m=-1, l=n-2)$ в силу (10) и (17) получаем

$$
\int_{0}^{1}(1-\eta)^{n-m-3-j} \omega^{(n-m-2)}(\eta) d \eta=0, \quad j=0, \ldots, n-l-3
$$

Если $n-l-4-j \geqslant 0$, то на основании формулы интегрирования по частям из (18) следуют равенства

$$
\int_{0}^{1} \eta(1-\eta)^{n-m-3-j} \omega^{(n-m-1)}(\eta) d \eta=0, \quad j=0, \ldots, n-l-4 .
$$

В случае $n-m-l-3 \geqslant 0$ аналогичным образом последовательно получаются равенства

$$
\int_{0}^{1} \eta^{s}(1-\eta)^{n-m-3-j} \omega^{(n-m-2+s)}(\eta) d \eta=0, \quad s=0, \ldots, m_{1}, \quad j=0, \ldots, n-l-3-s,
$$

где

$$
m_{1}= \begin{cases}m, & \text { если } n-m-l-3=0, \\ m+1, & \text { если } n-m-l-4 \geqslant 0 .\end{cases}
$$

В частности, при $n-m-l-4 \geqslant 0$ в силу (19) справедливы равенства

$$
\int_{0}^{1} \eta^{m+1}(1-\eta)^{l+1+i} \omega^{(n-1)}(\eta) d \eta=0, \quad i=0, \ldots, n-m-l-4,
$$

из которых следует, что

$$
\int_{0}^{1} \eta^{m+1+i}(\eta-1)^{l+1} \omega^{(n-1)}(\eta) d \eta=0, \quad i=0, \ldots, n-m-l-4
$$

или с учетом (16)

$$
\int_{0}^{1} \eta^{m+1+i}(\eta-1)^{l+1}\left|P_{n-1}(\eta)\right|^{p-1} \operatorname{sign} P_{n-1}(\eta) d \eta=0, \quad i=0, \ldots, n-m-l-4
$$


Поскольку условия (21) для $1<p<\infty$ однозначно [21, с. 50] определяют многочлен $Q_{n-1, p, m, l}(x)$, наименее уклоняющийся от нуля в метрике $L_{p}(0,1)$, то в силу $(16)$

$$
\omega^{(n-1)}(\xi)=\alpha\left|Q_{n-1, p, m, l}(\xi)\right|^{p-1} \operatorname{sign} Q_{n-1, p, m, l}(\xi) .
$$

Для определения коэффициента $\alpha$ воспользуемся условием (11).

Из (11) и (17) после перестановки порядка интегрирования имеем

$$
1=\int_{0}^{1} \omega(t) d t=\frac{1}{(n-m-2) !} \int_{0}^{1}(1-\eta)^{n-m-2} \omega^{(n-m-2)}(\eta) d \eta
$$

Используя формулу интегрирования по частям и (19), для $m \geqslant 0$ получаем

$$
\begin{aligned}
(n- & m-1+i) \int_{0}^{1} \eta^{i}(1-\eta)^{n-m-2} \omega^{(n-m-2+i)}(\eta) d \eta \\
& =-\int_{0}^{1} \eta^{i+1}(1-\eta)^{n-m-2} \omega^{(n-m-1+i)}(\eta) d \eta, \quad i=0, \ldots, m .
\end{aligned}
$$

Из (20)-(24) следует, что

$$
\begin{aligned}
1 & =\frac{(-1)^{n-1}}{(n-1) !} \int_{0}^{1} \eta^{m+1}(\eta-1)^{n-m-2} \omega^{(n-1)}(\eta) d \eta \\
& =\frac{(-1)^{n-1} \alpha}{(n-1) !} \int_{0}^{1} \eta^{n-l-2}(\eta-1)^{l+1}\left|Q_{n-1, p, m, l}(\eta)\right|^{p-1} \operatorname{sign} Q_{n-1, p, m, l}(\eta) d \eta \\
& =\frac{(-1)^{n-1} \alpha}{(n-1) !} \int_{0}^{1} Q_{n-1, p, m, l}(\eta)\left|Q_{n-1, p, m, l}(\eta)\right|^{p-1} \operatorname{sign} Q_{n-1, p, m, l}(\eta) d \eta \\
& =\frac{(-1)^{n-1} \alpha}{(n-1) !} \int_{0}^{1}\left|Q_{n-1, p, m, l}(\eta)\right|^{p} d \eta .
\end{aligned}
$$

Отсюда, выражая $\alpha$, на основании (22) получаем

$$
\omega^{(n-1)}(\xi)=\frac{(-1)^{n-1}(n-1) !\left|Q_{n-1, p, m, l}(\xi)\right|^{p-1} \operatorname{sign} Q_{n-1, p, m, l}(\xi)}{\int_{0}^{1}\left|Q_{n-1, p, m, l}(\eta)\right|^{p} d \eta}
$$

и

$$
\left\|\omega^{(n-1)}\right\|_{p^{\prime}}=\frac{(n-1) !}{\left\|Q_{n-1, p, m, l}\right\|_{p}} .
$$

В силу (14) и (15) заключаем, что

$$
A_{n, n-1, m, l}^{*} \leqslant\left\|\omega^{(n-1)}\right\|_{p^{\prime}} .
$$

Отсюда с учетом (7) и (26) получаем

$$
A_{n, n-1, m, l}^{*}=\frac{(n-1) !}{\left\|Q_{n-1, p, m, l}\right\|_{p}} .
$$

Отметим, что в случае $n-m-l-3=0$ (в частности, при $m=-1, l=n-2$ ) доказательство равенства (27) значительно упрощается. 
Согласно теореме Ролля из условий $(9),(10)$ следует, что у $\omega^{(n-1)}(\xi)$ существует не менее $n-m-l-3$ различных нулей на $(0,1)$. С другой стороны, из $(25)$ вытекает, что $\omega^{(n-1)}(\xi)$ имеет на $(0,1)$ не более $n-m-l-3$ различных нулей. Отсюда заключаем, что $\omega(\xi) \neq 0$ на $(0,1)$, и в силу $(11),(12)|\Lambda(x, \xi)| \leqslant 1$. Тогда на основании (14) получаем для $B_{n, n-1, m, l}^{*}$ оценку сверху

$$
B_{n, n-1, m, l}^{*} \leqslant\left\|B_{\omega}\right\|_{L_{r} \rightarrow L_{q}} \leqslant 1 .
$$

Для получения нижней оценки воспользуемся очевидньми неравенствами

$$
\begin{gathered}
\left\|Q_{n, p, m, l}\right\|_{p} \leqslant\left\|\left(x-\frac{1}{2}\right) Q_{n-1, p, m, l}(x)\right\|_{p} \leqslant \frac{1}{2}\left\|Q_{n-1, p, m, l}\right\|_{p}, \\
\left\|Q_{n, p, m, l}^{(n-1)}\right\|_{q}=n !\left\|x+\frac{a_{n-m-l-3}}{n}\right\|_{q} \geqslant n !\left\|Q_{1, q}\right\|_{q}=\frac{n !}{2(1+q)^{1 / q}} .
\end{gathered}
$$

Полагая $f(x)=Q_{n, p, m, l}(x)$ в неравенстве $(4)$, из $(27),(29)$ и (30) получаем, что

$$
\frac{n !}{2(1+q)^{1 / q}} \leqslant \frac{(n-1) !}{2}+n ! B_{n, n-1, m, l}^{*} .
$$

Отсюда следует неравенство

$$
B_{n, n-1, m, l}^{*} \geqslant \frac{1}{2}\left((1+q)^{-1 / q}-\frac{1}{n}\right) .
$$

Тем самьм, теорема доказана при $1<p<\infty$.

Рассмотрим теперь крайние случаи $p=1$ и $p=\infty$. Поскольку (см. [22, с. 128]) для $\delta \in\{1 ; \infty\}$

$$
\lim _{p \rightarrow \delta}\left\|Q_{n-1, p, m, l}\right\|_{p}=\left\|Q_{n-1, \delta, m, l}\right\|_{\delta}
$$

то, совершая предельный переход в $(27),(28)$ и (31), получаем (6) и для случая $p=\delta$. Теорема доказана.

СлЕДСТВИЕ 1. Пусть $1 \leqslant p, q, r \leqslant \infty u$ выполняются условия (5). Тогда

$$
A_{n, n-1, n-l-3, l}^{*}= \begin{cases}(n-1) !(\mathrm{B}(p(n-l-2)+1, p(l+1)+1))^{-1 / p}, & 1 \leqslant p<\infty \\ (n-1) !(n-1)^{n-1}(n-l-2)^{2+l-n}(l+1)^{-l-1}, & p=\infty\end{cases}
$$

где $\mathrm{B}(u, v)-$ бэта-функиия.

ДокАЗАТЕЛЬСТво. Достаточно заметить, что при $n-m-l-3=0$

$$
Q_{n-1, p, n-l-3, l}(x)=x^{n-l-2}(x-1)^{l+1} .
$$

СлЕДСТВИЕ 2. Пусть $1 \leqslant p \leqslant \infty, q=\infty, r=1, m=-1$ и выполняются условия (5). Тогда

$$
B_{n, n-1,-1, l}^{*}(p, \infty, 1)=1 \text {. }
$$


ДокАЗАТЕЛЬСТво. Для $q=\infty, r=1, k>n$ подставим в неравенство (4) функцию $f(x)=(x-1)^{k}$ и перейдем к пределу при $k \rightarrow \infty$. Тогда получим $B_{n, n-1,-1, l}^{*} \geqslant 1$, что в совокупности с (6) приводит к (32).

\section{2. Наименее уклоняющиеся от нуля многочлены.}

Теорема 2. Если $p=\infty$, то в случае $n \geqslant 2, m=-1, l=0$

$$
\begin{gathered}
Q_{n-1, \infty,-1,0}(x)=\left(2 \cos ^{2} \frac{\pi}{4(n-1)}\right)^{1-n} T_{n-1}\left(2 \cos ^{2} \frac{\pi}{4(n-1)} x-1\right) \\
\left\|Q_{n-1, \infty,-1,0}\right\|_{\infty}=2\left(4 \cos ^{2} \frac{\pi}{4(n-1)}\right)^{1-n}
\end{gathered}
$$

а в случае $n \geqslant 3, m=l=0$

$$
\begin{gathered}
Q_{n-1, \infty, 0,0}(x)=\left(2 \cos \frac{\pi}{2(n-1)}\right)^{1-n} T_{n-1}\left(\cos \frac{\pi}{2(n-1)}(2 x-1)\right) \\
\left\|Q_{n-1, \infty, 0,0}\right\|_{\infty}=2\left(4 \cos \frac{\pi}{2(n-1)}\right)^{1-n}
\end{gathered}
$$

где $T_{n-1}(x)=2^{2-n} \cos (n-1) \arccos x-$ многочлен Чебишева первого рода.

ДокАЗАТЕЛЬСтво. Приведем доказательство только первого утверждения теоремы (для второго утверждения доказательство аналогично).

Предположим, что многочлен

$$
P_{n-1}(x)=\left(2 \cos ^{2} \frac{\pi}{4(n-1)}\right)^{1-n} T_{n-1}\left(2 \cos ^{2} \frac{\pi}{4(n-1)} x-1\right)
$$

не является многочленом наименьшего уклонения от нуля в метрике $L_{\infty}(0,1)$, т.е. $\left\|Q_{n-1, \infty,-1,0}\right\|_{\infty}<\left\|P_{n-1}\right\|_{\infty}$. Тогда разность многочленов $P_{n-1}(x)-Q_{n-1, \infty,-1,0}(x)$ на отрезке $[0,1]$ имеет по крайней мере $n-1$ различных нулей, так как $Q_{n-1, \infty,-1,0}(1)=$ $P_{n-1}(1)=0$, и в силу свойств многочлена Чебьшева первого рода функция $P_{n-1}(x)$ на $[0,1)$ имеет $n-1$ точек альтернанса, в которых $P_{n-1}(x)$ принимает значение $\left\|P_{n-1}\right\|_{\infty} \mathrm{c}$ чередующимися знаками. Но это противоречит тому, что разность $P_{n-1}-Q_{n-1, \infty,-1,0}$ является полиномом степени не вьше $n-2$. Теорема доказана.

СлЕДСТВИЕ 3 . ДЛя любы $1 \leqslant q, r \leqslant \infty$ в случае $n \geqslant 2$

$$
A_{n, n-1,-1,0}^{*}(\infty, q, r)=\frac{(n-1) !}{2}\left(4 \cos ^{2} \frac{\pi}{4(n-1)}\right)^{n-1}
$$

а в случае $n \geqslant 3$

$$
A_{n, n-1,0,0}^{*}(\infty, q, r)=\frac{(n-1) !}{2}\left(4 \cos \frac{\pi}{2(n-1)}\right)^{n-1} .
$$


ДокАЗАТЕЛЬСтво. Для $m \in\{-1,0\}$ утверждения следствия вытекают из теорем 1 и 2.

Tеорема 3. Если $p=2, n \geqslant 3 u-1 \leqslant m+l \leqslant n-4, m o$

$$
\begin{gathered}
Q_{n-1,2, m, l}(x)=\frac{(n+m+l+1) !\left(x^{n+m-l-1}(x-1)^{n+l-m-1}\right)^{(n-m-l-3)}}{(2 n-2) ! x^{m+1}(x-1)^{l+1}}, \\
\left\|Q_{n-1,2, m, l}\right\|_{2}=\sqrt{\frac{(n+m+l+1) !(n-m-l-3) !(n+m-l-1) !(n+l-m-1) !}{(2 n-2) !(2 n-1) !}} .
\end{gathered}
$$

ДокАЗАТЕЛЬСТво. Функция $\Phi_{2}(b)$ является дифференцируемой и выпуклой. Следовательно, в экстремальной задаче $\Phi_{2}(b) \rightarrow \inf$ необходимым и достаточным условием минимума $[21$, с. 50$]$ являются соотношения

$$
\int_{0}^{1} x^{i+m+1}(x-1)^{l+1} Q_{n-1,2, m, l}(x) d x=0, \quad i=0, \ldots, n-m-l-4 .
$$

Легко проверить, что соотношения (34) вьполняются для многочлена $Q_{n-1,2, m, l}(x)$, определенного в (33).

Используя (34) и формулу интегрирования по частям, получаем

$$
\begin{aligned}
\left\|Q_{n-1,2, m, l}\right\|_{2}^{2} & =\int_{0}^{1} x^{n-l-2}(x-1)^{l+1} Q_{n-1,2, m, l}(x) d x \\
& =\frac{(n+m+l+1) !}{(2 n-2) !}(n+m-l-3) ! \mathrm{B}(n+m-l, n+l-m) .
\end{aligned}
$$

Теорема доказана.

СледСтвиЕ 4 . Для любы $1 \leqslant q, r \leqslant \infty$ в случае $n \geqslant 3 u-1 \leqslant m+l \leqslant n-4$

$$
\begin{aligned}
& A_{n, n-1, m, l}^{*}(2, q, r) \\
& \quad=(n-1) ! \sqrt{\frac{(2 n-2) !(2 n-1) !}{(n+m+l+1) !(n-m-l-3) !(n+m-l-1) !(n+l-m-1) !}} .
\end{aligned}
$$

ДокАЗАТЕЛЬСТво. На основании теорем 1 и 3 получаем утверждение следствия.

ЛЕмма. Пусть

$$
\mathrm{B}_{c}(a, b)=\int_{c}^{1} t^{a-1}(1-t)^{b-1} d t
$$

где $b>0, a+b \neq 1$ и $a>0$ при $c=0$ или $a \neq 0$ при $0<c \leqslant 1$. Тогда

$$
\begin{gathered}
(a+b-1) \mathrm{B}_{c}(a, b)=-c^{a}(1-c)^{b-1}+(b-1) \mathrm{B}_{c}(a, b-1) \\
(a+b-1) \mathrm{B}_{c}(a, b)=c^{a-1}(1-c)^{b}+(a-1) \mathrm{B}_{c}(a-1, b)
\end{gathered}
$$


ДОКАЗАТЕЛЬСТво. ИспользУя тождество

$$
t^{a}(1-t)^{b-2}=t^{a-1}(1-t)^{b-2}-t^{a-1}(1-t)^{b-1}
$$

и формулу интегрирования по частям, получаем

$$
\mathrm{B}_{c}(a, b)=-a^{-1}\left(c^{a}(1-c)^{b-1}-(b-1) \mathrm{B}_{c}(a, b-1)+(b-1) \mathrm{B}_{c}(a, b)\right) .
$$

Отсюда следует (35).

Используя тождество

$$
t^{a-2}(1-t)^{b}=(1-t)^{b-1} t^{a-2}-(1-t)^{b-1} t^{a-1}
$$

и формулу интегрирования по частям, получаем

$$
\mathrm{B}_{c}(a, b)=b^{-1}\left(c^{a-1}(1-c)^{b}+(a-1) \mathrm{B}_{c}(a-1, b)+(a-1) \mathrm{B}_{c}(a, b)\right) .
$$

Отсюда следует (36). Лемма доказана.

Отметим, что $\mathrm{B}_{0}(a, b)$ является обычной бэта-функцией $\mathrm{B}(a, b)$.

Teорема 4. Пусть $1 \leqslant p \leqslant \infty, n \geqslant 3, m=-1 u l=n-3$. Тогда

$$
\begin{gathered}
Q_{n-1, p,-1, n-3}(x)=(x-1)^{n-2}\left(x-1+a_{p}\right), \\
\left\|Q_{n-1, p,-1, n-3}\right\|_{p}=\left(1-a_{p}\right)(1+(n-1) p)^{-1 / p},
\end{gathered}
$$

где $a_{p} \in(0,1)$ является единственным корнем уравнения

$$
\begin{aligned}
\mathrm{B}_{a_{p}}(p(1-n), p)-\mathrm{B}(p(n-2)+1, p)=0, & \text { еслu } 1 \leqslant p<\infty \\
(n-2)^{n-2}(n-1)^{1-n} a_{p}^{n-1}+a_{p}-1=0, & \text { если } p=\infty .
\end{aligned}
$$

ДокАЗАТЕЛЬСтво. Исследуем сначала случай $1 \leqslant p<\infty$. Поскольку функция

$$
F(a)=\int_{0}^{1}\left|(x-1)^{n-2}(x-1+a)\right|^{p} d x=\int_{0}^{1}\left|x^{n-2}(x-a)\right|^{p} d x
$$

дифференцируема, то для многочлена $Q_{n-1, p,-1, n-3}(x)=(x-1)^{n-2}\left(x-1+a_{p}\right)$, наименее уклоняюшегося от нуля в метрике $L_{p}(0,1)$, коэффициент $a_{p}$ необходимо является корнем уравнения $F^{\prime}\left(a_{p}\right)=0$, что эквивалентно

$$
\int_{0}^{1} x^{n-2}\left|x^{n-1}-a_{p} x^{n-2}\right|^{p-1} \operatorname{sign}\left(x^{n-1}-a_{p} x^{n-2}\right) d x=0 .
$$

Из (37) следует, что $0<a_{p}<1$. Равенство (37) эквивалентно равенству

$$
\int_{a_{p}}^{1} x^{p(n-2)}\left(x-a_{p}\right)^{p-1} d x-\int_{0}^{a_{p}} x^{p(n-2)}\left(a_{p}-x\right)^{p-1} d x=0
$$


или после несложных преобразований

$$
\mathrm{B}_{a_{p}}(p(1-n), p)-\mathrm{B}(p(n-2)+1, p)=0
$$

Единственность $a_{p}$ следует из того, что левая часть равенства (38) монотонно убывает по $a_{p}$ на интервале $(0,1)$.

При вычислении нормы аналогично получаем

$$
\left\|Q_{n-1, p,-1, n-3}\right\|_{p}^{p}=a_{p}^{p(n-1)+1}\left(\mathrm{~B}(p(n-2)+1, p+1)+\mathrm{B}_{a_{p}}(p(1-n)-1, p+1)\right) .
$$

Отсюда, используя лемму и (38), имеем

$$
\begin{aligned}
\left\|Q_{n-1, p,-1, n-3}\right\|_{p}^{p}= & \frac{a_{p}^{p(n-1)+1}}{1+p(n-1)}\left(a_{p}^{p(1-n)}\left(1-a_{p}\right)^{p}+a_{p}^{p(1-n)-1}\left(1-a_{p}\right)^{p+1}\right. \\
& \left.+p\left(\mathrm{~B}(p(n-2)+1, p)-\mathrm{B}_{a_{p}}(p(1-n), p)\right)\right) \\
= & \frac{\left(1-a_{p}\right)^{p}}{1+p(n-1)} .
\end{aligned}
$$

В случае $p=\infty$ уравнение

$$
(n-2)^{n-2}(n-1)^{1-n} a_{p}^{n-1}=1-a_{p}
$$

имеет единственньй корень $a_{p} \in(0,1)$, для которого многочлен

$$
P_{n-1}(x)=(x-1)^{n-2}\left(x-1+a_{p}\right)
$$

в точках $x=0$ и $x=1-(n-2)(n-1)^{-1} a_{p}$ принимает значение $\left\|P_{n-1}\right\|_{\infty}=1-a_{p}$ с противоположньми знаками. Если предположить, что $P_{n-1}(x)$ не является наименее уклоняющимся от нуля многочленом, то $\left\|P_{n-1}\right\|_{\infty}>\left\|Q_{n-1, \infty,-1, n-3}\right\|_{\infty}$ и разность $P_{n-1}(x)-Q_{n-1, \infty,-1, n-3}(x)$ имеет нуль на отрезке $\left[0,1-(n-2)(n-1)^{-1} a_{p}\right]$, что невозможно, так как $P_{n-1}(x)-Q_{n-1, \infty,-1, n-3}(x)=c(x-1)^{n-2}$. Следовательно, $P_{n-1}(x) \equiv Q_{n-1, \infty,-1, n-3}(x)$. Теорема доказана.

СлеДСТВИЕ 5. Для любы $1 \leqslant p, q, r \leqslant \infty$ в случае $n \geqslant 3, m=-1 u 0 \leqslant l \leqslant n-3$

$$
A_{n, n-1,-1, n-3}^{*}=(n-1) !\left(1-a_{p}\right)^{-1}(1+(n-1) p)^{1 / p},
$$

где $a_{p}$ определяется как в теореме 4.

ДокАЗАТЕЛьство. Утверждение следствия вытекает из теорем 1 и 4.

СлЕДСТВИЕ 6 . Пусть $1 \leqslant p, q, r \leqslant \infty u$ выполняются условия (5). Тогда

$$
\frac{(n-1) !(n-1)^{n-1}}{(n-l-2)^{n-l-2}(l+1)^{l+1}} \leqslant A_{n, n-1, m, l}^{*} \leqslant(n-1) ! 4^{n-1} \text {. }
$$


ДокАЗАТЕЛЬСТво. Оценки для $A_{n, n-1, m, l}^{*}$ следуют из теоремы 1, следствия 1 и очевидных неравенств

$$
\begin{aligned}
\left\|Q_{n-1,1}\right\|_{1} & =\left\|Q_{n-1,1,-1,-1}\right\|_{1} \leqslant\left\|Q_{n-1,1, m, l}\right\|_{1} \leqslant\left\|Q_{n-1, p, m, l}\right\|_{1} \leqslant\left\|Q_{n-1, p, m, l}\right\|_{p} \\
& \leqslant\left\|Q_{n-1, p, n-l-3, l}\right\|_{p} \leqslant\left\|Q_{n-1, \infty, n-l-3, l}\right\|_{p} \leqslant\left\|Q_{n-1, \infty, n-l-3, l}\right\|_{\infty},
\end{aligned}
$$

где $Q_{n-1,1}(x)=2^{1-n} U_{n-1}(2 x-1), U_{n-1}(x)=2^{1-n}\left(1-x^{2}\right)^{-1 / 2} \sin n \arccos x-$ многочлен Чебышева второго рода.

\section{3. Неравенство с двумя точными константами.}

Teоpema 5. Пусть $n \in\{3,4, \ldots\}, m \in\{0, \ldots, n-3\}, 1 \leqslant p \leqslant \infty, M_{0}, M_{n-1}, M_{n} \geqslant 0$. Тогда для того чтоби существовала функиия $f$ такая, что

$$
\begin{gathered}
f(0)=f^{\prime}(0)=\cdots=f^{(m)}(0)=0 \\
\|f\|_{p}=M_{0}, \quad\left\|f^{(n-1)}\right\|_{\infty}=M_{n-1}, \quad\left\|f^{(n)}\right\|_{1}=M_{n}
\end{gathered}
$$

необходимо и достаточно, чтоби

1) если $M_{0}=0$, mо $M_{n-1}=M_{n}=0$;

2) если $M_{0}>0$ u $M_{n-1}=0$, то $M_{n}=0$;

3) если $M_{0}>0, M_{n-1}>0 u M_{n}=0$, mo

$$
M_{n-1} \leqslant(n-1) !\left\|Q_{n-1, p, m,-1}\right\|_{p}^{-1} M_{0}
$$

4) если $M_{0}>0, M_{n-1}>0, M_{n}>0$, mo

$$
M_{n-1}<(n-1) !\left\|Q_{n-1, p, m,-1}\right\|_{p}^{-1} M_{0}+M_{n}
$$

ДокАЗАТЕльство. Случаи 1) и 2) очевидны. В случае 3) необходимость следует из теоремы 1. Для доказательства достаточности условия (40) рассмотрим функцию

$$
f_{c}(x)=\frac{M_{n-1}}{(n-1) !} Q_{n-1, p, m,-1}(x)+c x^{n-2},
$$

которая при произвольном $c \geqslant 0$ удовлетворяет равенствам (39) и

$$
\left\|f_{c}^{(n-1)}\right\|_{\infty}=M_{n-1}, \quad\left\|f_{c}^{(n)}\right\|_{1}=0
$$

Поскольку в силу (40) $\left\|f_{0}\right\|_{p} \leqslant M_{0}$, a $\left\|f_{c}\right\|_{p}$ непрерывно зависит от $c \geqslant 0$ и неограниченно возрастает при $c \rightarrow \infty$, то существует $c_{0}$, для которого $\left\|f_{c_{0}}\right\|_{p}=M_{0}$.

В случае 4) необходимость следует из теоремы 1 и следствия 2 , если дополнительно учесть $[2$, с. 46$]$, что

$$
\left\|\int_{0}^{1} f^{(n)}(\xi) \Lambda(x, \xi) d \xi\right\|_{\infty}<\left\|f^{(n)}\right\|_{1},
$$

когда $\left\|f^{(n)}\right\|_{1}>0$. 
Для доказательства достаточности в случае 4) исследуем сначала частньй подслучай $M_{n} \leqslant M_{n-1}$. Определим функцию

$$
f_{c}(x)=\frac{M_{n-1}-M_{n}}{(n-1) !} Q_{n-1, p, m,-1}(x)+c x^{n-2}+\frac{1}{(n-2) !} \int_{0}^{1}(x-t)_{+}^{n-2} w_{\varepsilon}(t) d t
$$

где $\varepsilon=\min \{c, 1 / 2\}, c>0$ и

$$
w_{\varepsilon}(t)= \begin{cases}\varepsilon^{-1} M_{n}(\varepsilon-t), & 0 \leqslant t \leqslant \varepsilon \\ 0, & \varepsilon<t \leqslant 1\end{cases}
$$

Для любого $c>0$ функция $f_{c}(x)$ удовлетворяет условиям $(39), f_{c}^{(n-1)}(x)=M_{n-1}-$ $M_{n}+w_{\varepsilon}(x)$ и

$$
\left\|f_{c}^{(n-1)}\right\|_{\infty}=M_{n-1}, \quad\left\|f_{c}^{(n)}\right\|_{1}=M_{n}
$$

Поскольку

$$
\left|\int_{0}^{1}(x-t)_{+}^{n-2} w_{\varepsilon}(t) d t\right| \leqslant \frac{\varepsilon}{2} M_{n}
$$

то с учетом (41)

$$
\lim _{c \rightarrow+0}\left\|f_{c}\right\|_{p}=\frac{M_{n-1}-M_{n}}{(n-1) !}\left\|Q_{n-1, p, m,-1}\right\|_{p}<M_{0}
$$

Тогда в силу непрерывной зависимости $\left\|f_{c}\right\|_{p}$ от $c>0$ и свойства

$$
\lim _{c \rightarrow+\infty}\left\|f_{c}\right\|_{p}=+\infty
$$

найдется $c_{0}$ такое, что $\left\|f_{c_{0}}\right\|_{p}=M_{0}$.

В подслучае $M_{n-1} \leqslant M_{n}$ воспользуемся построенной Буренковым $[2$, с. 46] функцией $\varphi(x) \geqslant 0$, для которой справедливы свойства

$$
\int_{0}^{1} \varphi(x) d x<M_{0}, \quad\|\varphi\|_{\infty}=M_{n-1}, \quad\left\|\varphi^{\prime}\right\|_{1}=M_{n} .
$$

Рассмотрим удовлетворяющую условиям (39) функцию

$$
f_{c}(x)=c x^{n-2}+\frac{1}{(n-2) !} \int_{0}^{1}(x-t)_{+}^{n-2} \varphi(t) d t,
$$

для которой при любом $c \geqslant 0$

$$
\left\|f_{c}^{(n-1)}\right\|_{\infty}=\|\varphi\|_{\infty}=M_{n-1}, \quad\left\|f_{c}^{(n)}\right\|_{1}=\left\|\varphi^{\prime}\right\|_{1}=M_{n} .
$$

Кроме того,

$$
\left\|f_{0}\right\|_{p} \leqslant\left\|f_{0}\right\|_{\infty} \leqslant \frac{1}{(n-2) !} \int_{0}^{1} \varphi(t) d t<M_{0}, \quad \lim _{c \rightarrow+\infty}\left\|f_{c}\right\|_{p}=+\infty .
$$

Следовательно, можно подобрать $c_{0}$ так, что $\left\|f_{c_{0}}\right\|_{p}=M_{0}$. Теорема доказана.

Выражаю благодарность профессору В.И. Буренкову за постановку задачи. 


\section{СПИСОК ЦИТИРОВАННОЙ ЛИТЕРАТУРЫ}

[1] Буренков В.И. О точных постоянных в неравенствах для норм промежуточных производных на конечном интервале // Тр. МИАН. 1980. Т. 156. С. 22-29.

[2] Буренков В.И. О точных постоянных в неравенствах для норм промежуточных производных на конечном интервале. II // Тр. МИАН. 1986. Т. 173. С. 38-49.

[3] Landau E. Einige Ungleichungen für zweimal differetierbare Funktionen // Proc. London Math. Soc. 1913. V. 13. P. 43-49.

[4] Hadamard J. Sur le module maximum d'une fonction et de ses derivées // C. R. Acad. Sci. Paris. Sér. I. Math. 1914. V. 41. P. 68-72.

[5] Звягинцев А. И. Некоторые оценки для норм функции и ее производных на конечном интервале // Латвийский матем. ежегодник. 1985. № 29. С. 198-210.

[6] Звягинцев А. И. Оценки для промежуточной производной функции // Латвийский матем. ежегодник. 1988. №32. С. 183-186.

[7] Гусаков В. А. Неравенства с точными постоянными для норм промежуточных производных. Дисс. ... к. ф.-м. н. М.: Университет Дружбы народов, 1990.

[8] Kallioniemi H. The Landau problem on compact intervals and optimal numerical differentiation // J. Approx. Theory. 1990. V. 63. P. 72-91.

[9] Шадрин А. Ю. О точных постоянных в неравенствах между $L_{\infty}$-нормами производных на конечном отрезке // Докл. РАН. 1992. Т. 326. №1. С. 50-53.

[10] Шадрин А. Ю. Интерполяция многочленами Лагранжа, точные постоянные в неравенствах между нормами производных на конечном отрезке // Матем. заметки. 1993. Т. 54. № 5. C. 129-143.

[11] Boyd D. Best constants in a class of integral inequalities // Pacific J. Math. 1969. V. 30. № 2. P. 367-383.

[12] Bohl P. Über Differentialungleichungen // J. Reine Angew. Math. 1913. V. 144. P. 284-313.

[13] Tumura M. Kôkai zyôbibunhôteisiki ni tuite // Kansû Hôteisiki. 1941. V. 30. P. 20-35.

[14] Fan K., Taussky O., Todd J. Discrete analogs of inequalities of Wirtinger // Monatsh. Math. 1955. V. 59. №2. P. 73-90.

[15] Hukuhara M. On the zeros of solutions of linear ordinary differential equations // Sugaku. 1963. V. 15. P. 108-109.

[16] Brink J. Inequalities involving $\|f\|_{p}$ and $\left\|f^{(n)}\right\|_{q}$ for $f$ with $n$ zeros // Pacific J. Math. 1972. V. 42. № 2. P. 289-311.

[17] Кигурадзе И. Т. Некоторые сингулярные краевые задачи для обыкновенных дифференциальных уравнений. Тбилиси: Изд-во ТГУ, 1975.

[18] Габушин В. Н. Неравенства между производными в метриках $L_{p}$ при $0<p \leqslant \infty / /$ Изв. АН СССР. Сер. матем. 1976. Т. 40. № 4. С. 869-892.

[19] Буслаев А. П. О точных константах и экстремальных функциях в неравенствах для производных // Матем. заметки. 1987. Т. 41. № 2. С. 159-174.

[20] Айунц С. А. Совершенные $\Gamma_{0}$-сплайны и точечная задача Колмогорова-Ландау. Деп. АрмНИИНТИ 23.05.89. № 23-Ар89. Ереван: ЕГУ, 1989.

[21] Тихомиров В. М. Некоторые вопросы теории приближений. М.: Изд-во МГУ, 1976.

[22] Никольский С. М. Квадратурные формулы. М.: Наука, 1974.

Высшая административная школа при мэрии Санкт-Петербурга

Поступило 26.03.96

Исправленный вариант 08.04 .96 